\title{
Desempenho, temperatura retal e freqüência respiratória de novilhas leiteiras de três grupos genéticos recebendo dietas com diferentes níveis de fibra ${ }^{1}$
}

\author{
José Carlos Pereira², Daniel de Noronha Figueiredo Vieira da Cunha ${ }^{3}$, Paulo Roberto \\ Cecon ${ }^{4}$, Elisa de Souza Faria ${ }^{3}$
}

\footnotetext{
1 Projeto financiado pela FAPEMIG.

2 Departamento de Zootecnia - UFV. Pesquisador do CNPq.

3 Programa de Pós-graduação em Zootecnia - UFV - Viçosa, MG.

${ }^{4}$ Departamento de Informática - UFV.
}

RESUMO - Foram avaliados o desempenho, a temperatura retal e a freqüência respiratória de novilhas leiteiras de três grupos genéticos, Holandês $(\mathrm{H}) \times$ Zebu (Z) (7/8 HZ, 15/16 HZ e Holandês puro por cruza - HPC), mantidas em sistema de confinamento e recebendo dietas com diferentes níveis de fibra em detergente neutro (30 e 60\%) utilizando-se como volumoso capim-elefante (Pennisetum purpureum, Schum., cv. Mineirão) picado. Determinaram-se o consumo, o ganho de peso e as respostas fisiológicas desses animais considerando as condições climáticas nos dias experimentais. O experimento foi delineado segundo esquema fatorial $3 \times 2$ (grupo genético $\times$ nível de fibra), em blocos casualizados, com quatro repetições. Os valores relativos às variáveis fisiológicas foram analisados em parcelas subdivididas no tempo. Os consumos médios de MS, FDN e FDA foram de 10,13; 2,69 e 1,45 kg/dia e de 2,70; 0,71 e 0,38\%PV, respectivamente, para a dieta com baixa fibra e de 10,68; 6,17 e 2,92 kg/dia e 2,90; 1,67 e 0,79\%PV para a dieta com alta fibra para ganhos de peso diários de 1,28 e 0,92 kg/dia, respectivamente. O consumo de MS em \%PV pelos animais HPC foi maior (2,94\%PV) que pelos animais 7/8HZ (2,65\%PV). As novilhas que receberam a dieta com $60 \%$ de FDN consumiram maior quantidade de MS, FDN e FDA e apresentaram menores ganhos de peso por dia em relação àquelas que receberam a dieta com 30\% de FDN. Novilhas HPC consomem maior quantidade de MS (\%PV) que as 7/8 HZ, tanto em dietas com 30\% de FDN quanto com 60\% de FDN. Nas condições de ambiente térmico observadas durante o período experimental, novilhas 7/8 HZ, 15/16 HZ e HPC mantêm a temperatura retal dentro de limites fisiológicos considerados normais.

Palavras-chave: consumo, ganho de peso, índices fisiológicos

\section{Performance, rectal temperature and respiratory ratio of dairy heifers from three genetic groups fed diets with different levels of fiber}

\begin{abstract}
This work aimed to evaluate the performance, rectal temperature and respiratory ratio of dairy heifers, from three genetic groups Holstein $\times$ Zebu $(7 / 8 \mathrm{HZ}, 15 / 16 \mathrm{HZ}$, Graded Holstein-GH), confined and fed total mixed diet with different levels of neutral detergent fiber (30 and 60\%), using chopped elephantgrass (Pennisetum purpureum, Schum., cv. Mineirão) as forage. Intake, weight gain and physiological responses of these animals were determined under different environmental conditions. The experiment was conducted according to a $3 \times 2$ factorial arrangement (genetic group $\times$ fiber level), in a completely randomized design, with three replicates. The values related to physiological responses were analyzed according to split plot design in the time. Average intakes of DM, NDF and ADF of 10.13, 2.69, and 1.45 kg/day and 2.70, 0.71 and $0.38 \% \mathrm{LW}$, respectively, were observed for the diet with low fiber content versus 10.68, 6.17, and $2.92 \mathrm{~kg} / \mathrm{day}$ and 2.90,1.67, and $0.79 \% \mathrm{BW}$ for the diet with high fiber content and weight gains of 1.28 and $0.92 \mathrm{~kg} /$ day, respectively were obtained. The DM intake in \%BW for GH animals was higher (2.94 \%BW) than for animals 7/8HZ (2,65\%LW). When elephantgrass was used as forage only, heifers fed diets with $60 \%$ of NDF ingested greater amount of DM, NDF and ADF and gained less weight per day in relation to those fed diets with $30 \%$ of NDF. GH heifers ingested more DM (\%LW) than 7/8 HZ heifers, for both diets with 30 and 60\% NDF. In thermal environment conditions observed during the experimental period, heifers $7 / 8 \mathrm{HZ}, 15 / 16 \mathrm{HZ}$ and $\mathrm{GH}$ keep the rectal temperature inside the physiological limits considered normal.
\end{abstract}

Key Words: intake, physiological parameters, weight gain

Este artigo foi recebido em 6/4/2006 e aprovado em 31/8/2007.

Correspondências devem ser enviadas para jcarlos@ufv.br. 


\section{Introdução}

Os sistemas de determinação das exigências nutricionais de bovinos de rebanhos leiteiros são, em sua maioria, baseados em estudos realizados em regiões de clima temperado utilizando-se animais de raças européias e forrageiras adaptados às condições locais.

Como dois terços do território brasileiro localizam-se na faixa tropical, onde predominam altas temperaturas, elevada incidência de radiação solar, baixa amplitude térmica ao longo do ano e grande amplitude térmica diária (Titto, 1998), a aplicação de recomendações geradas em condições de clima temperado deve ser observada com ressalvas. Segundo West (2003), embora o NRC (2001) não considere os efeitos do estresse térmico sobre as exigências nutricionais de bovinos de rebanhos leiteiros, muitos autores já os demonstraram.

De acordo com Cardoso et al. (2000), quando fornecidas rações com alto teor de fibra, ou baixa densidade energética, o consumo é limitado pelo efeito de “enchimento" do rúmen retículo, no entanto, se a densidade energética é elevada ou a concentração de fibra é baixa, a ingestão passa a ser limitada pela demanda fisiológica de energia. Entretanto, em regiões tropicais, o consumo de matéria seca e o desempenho de bovinos pode ser reduzido, também, de acordo com a intensidade e a duração do estresse por calor (McDowell, 1972; Titto, 1998) ou mesmo pela interação densidade energética da ração $\times$ temperatura efetiva a que os animais estão submetidos.

As recomendações quanto aos níveis de FDN em rações para bovinos de rebanhos leiteiros são pontuais e se restringem a alimentos e categorias animais específicas. Segundo o NRC (2001), vacas leiteiras, quando alimentadas, em sistema de ração em mistura completa, com silagem de milho ou alfafa na ração total, podem tolerar dietas com $25 \%$ de FDN, desde que 19\% dessa fibra seja proveniente do volumoso.

Informações na literatura (Tomlinson et al., 1991) quanto aos níveis adequados de fibra em rações para novilhas de rebanhos leiteiros são escassas e bastante variáveis, principalmente quanto às relações entre o grupo genético, os níveis de fibra das rações e a termorregulação após a puberdade, fase importante na formação do animal adulto. Nesse sentido, objetivou-se com este estudo avaliar o desempenho, a temperatura retal e a freqüência respiratória de novilhas de três grupos genéticos mantidas em confinamento e alimentadas com rações contendo diferentes níveis de fibra utilizando-se capim-elefante (Pennisetum purpureum Schum., cv. Mineirão) como volumoso.

\section{Material e Métodos}

O experimento foi conduzido no Departamento de Zootecnia da Universidade Federal de Viçosa, localizada na região da Zona da Mata Mineira, latitude de $20^{\circ} 45^{\prime}$ S e $42^{\circ} 51$ 'N, GRW, com altitude média de $635 \mathrm{~m}$. A região apresenta clima subtropical do tipo Cwa - mesotérmico, segundo classificação de Köppen (1948), com verões quentes e invernos frios e secos (Tabela 1).

Foram utilizadas 24 novilhas não-gestantes, em arranjo fatorial $3 \times 2$ (três grupos genéticos $\times$ dois níveis de fibra na dieta) e delineamento em blocos casualizados, com quatro repetições. Os grupos genéticos foram obtidos pelo cruzamento entre animais da raça Holandesa Preto e Branco $(\mathrm{H})$ e animais Zebu (Z) com predominância da raça Gir: 7/8 HZ, 15/16 HZ e Holandês Puro por cruza (HPC). As novilhas possuíam pelagens semelhantes e não apresentavam problemas de ordem reprodutiva.

Os animais foram selecionados com base no peso corporal médio ( $375 \mathrm{~kg}$ ) e no escore de condição corporal, entre 3,0 e 3,5 em escala de 1 (muito magro) a 5 (obeso), e distribuídas aleatoriamente em baias individuais com piso concretado (12 $\mathrm{m}^{2}$ por animal) parcialmente sombreadas ( $6 \mathrm{~m}^{2}$ de sombra às $\left.12 \mathrm{~h}\right)$.

As dietas (Tabela 2) foram estabelecidas para conter dois níveis de fibra em detergente neutro (FDN): 30\% (FDN30) e 60\% (FDN60) na matéria seca (MS) da ração considerando as exigências nutricionais descritas pelo NRC (1989) para ganho médio de 700 g/animal/dia. As rações foram formuladas em mistura completa, contendo capim-elefante picado como volumoso. As dietas foram ajustadas a cada período experimental de acordo com a análise bromatológica prévia do volumoso.

A relação volumoso:concentrado, com base na MS, foi de 29:71 na dieta FDN30 e 50:50 na dieta FDN60. A ração foi fornecida duas vezes ao dia, às 10 e $16 \mathrm{~h}, 60 \%$ na parte da manhã e $40 \%$ no período da tarde. Para início do fornecimento das dietas, tomou-se como base o consumo voluntário inicial de MS, de 2,2\% do peso corporal, de acordo com o NRC (1989). Durante o período de adaptação, o consumo foi ajustado para $10 \%$ de sobras visando garantir ingestão à vontade da ração. $\mathrm{O}$ acesso irrestrito à água e à mistura mineral foi garantido diariamente para todos os animais.

Adotou-se um período inicial de 14 dias para ajuste do consumo e adaptação dos animais e, em seguida, decorreram-se três períodos experimentais de 14 dias cada um para as coletas dos dados. 
Tabela 1 - Precipitação pluviométrica (PP), radiação solar (RDSOL), umidade relativa (UR) e temperatura do ar ${ }^{1}$

\begin{tabular}{|c|c|c|c|c|c|c|}
\hline \multirow[t]{2}{*}{ Mês } & \multirow[t]{2}{*}{$\mathrm{PP}(\mathrm{mm})$} & \multirow[t]{2}{*}{ RDSOL (horas/dia) } & \multirow[t]{2}{*}{ UR (\%) } & \multicolumn{3}{|c|}{ Temperatura do ar $\left({ }^{\circ} \mathrm{C}\right)$} \\
\hline & & & & Média & Máxima & Mínima \\
\hline Setembro & 5,6 & 5,9 & 71,3 & 22,3 & 27,5 & 15,4 \\
\hline Outubro & 257,5 & 3,4 & 82,0 & 21,1 & 25,2 & 16,7 \\
\hline Novembro & 216,9 & 3,3 & 83,0 & 21,5 & 26,0 & 16,9 \\
\hline
\end{tabular}

${ }^{1}$ Setembro-outubro-novembro/1998.

Tabela 2 - Composição nutricional média dos ingredientes e das dietas experimentais (\% MS)

\begin{tabular}{lccrrrrr}
\hline Ingrediente & MS (\%) & FDN & FDA & LIG & CEL & PB & NDT $^{1}$ \\
\hline Capim-elefante & 22,86 & 78,15 & 45,91 & 5,05 & 38,25 & 7,95 \\
Farelo de soja & 86,30 & 12,82 & 7,72 & 0,27 & 7,25 & 56,53 & 53 \\
Farelo de trigo & 84,75 & 39,05 & 12,24 & 2,86 & 9,15 & 16,90 \\
Farelo de algodão & 87,83 & 46,36 & 30,51 & 14,00 & 12,00 & 29,69 & 70 \\
Fubá de milho & 86,93 & 11,29 & 2,64 & 0,07 & 2,75 & 9,98 & 85 \\
\hline FDN30 & 44,59 & 32,93 & 16,73 & 1,85 & 14,17 & 15,45 & 72 \\
FDN60 & 34,91 & 60,59 & 29,90 & 4,14 & 24,98 & 13,11 & 60 \\
\hline
\end{tabular}

$\mathrm{CEL}=$ celulose

${ }^{1}$ Valores obtidos em tabelas de composição de alimentos.

O consumo voluntário foi determinado pela pesagem diária da ração fornecida e das sobras. Diariamente, foram coletadas amostras do volumoso, da ração e das sobras. Amostras dos ingredientes e da mistura do concentrado foram coletadas no início, no meio e ao final de cada período experimental. Em todos os períodos, para as amostras compostas dos alimentos e das sobras de cada animal, foram determinados os teores de MS, proteína bruta (PB), fibra em detergente neutro (FDN), fibra em detergente ácido (FDA), lignina (LIG), hemicelulose (HEM) e celulose (CEL). A PB foi analisada de acordo com metodologia descrita por Silva \& Queiroz (2002). O método de análise seqüencial proposto por Van Soest et al. (1991) foi utilizado para determinação das frações FDN, FDA, lignina, celulose e hemicelulose.

Para caracterização do ambiente térmico, foram utilizados termohigrômetro, termômetro de globo negro e termômetros de máxima e mínima. Os valores mensurados pelo termômetro de globo negro e pelo termohigrômetro foram registrados, diariamente, às $7 \mathrm{~h}, 11 \mathrm{~h}, 15 \mathrm{~h}$ e $19 \mathrm{~h}$ e, semanalmente, de hora em hora para cálculo das estimativas médias diárias dos Índices de Temperatura e Umidade (ITU) e de Temperatura do Globo e Umidade (ITGU) e para avaliação da variação desses índices ao longo do dia. As temperaturas mensuradas pelos termômetros de máxima e mínima foram registradas diariamente às $7 \mathrm{~h}$ e às $19 \mathrm{~h}$. O ITU foi calculado segundo Baccari et al. (1998):

$$
\text { ITU }=\text { Tbs }+0,36 \text { Tpo }+41,2
$$

em que Tbs é a temperatura do termômetro de bulbo seco $\left({ }^{\circ} \mathrm{C}\right)$ e Tpo, a temperatura de ponto de orvalho $\left({ }^{\circ} \mathrm{C}\right)$. O ITGU foi calculado de acordo com Buffington et al. (1981):

$$
\text { ITGU }=0,72(\text { Tgn }+ \text { Tbu })+40,6
$$

em que Tgn representa a temperatura do termômetro de globo negro $\left({ }^{\circ} \mathrm{C}\right)$ e Tbu, a temperatura do termômetro de bulbo úmido $\left({ }^{\circ} \mathrm{C}\right)$.

Para avaliação das respostas fisiológicas das novilhas ao ambiente térmico, a freqüência respiratória e a temperatura retal foram mensuradas a cada três dias, às 9 e 15h. A freqüência respiratória, em número de respirações por minuto, foi estimada observando-se o flanco do animal durante 15 segundos e multiplicando-se o valor por quatro. A temperatura retal foi medida utilizando-se termômetro clínico veterinário inserido no reto dos animais durante 1 minuto e 30 segundos. A temperatura retal medida pela manhã foi subtraída do valor aferido à tarde para obtenção da diferença entre as duas medições, que representa a variação da temperatura retal entre os períodos da manhã e da tarde. As faixas de conforto, de risco e de estresse térmico nos animais foram determinadas com base nas observações de Du Preez et al. (1990a,b).

Os animais foram pesados no primeiro e no último dia de cada período, antes do fornecimento da ração da manhã. Os dados de consumo e ganho de peso foram submetidos à análise de variância e as médias foram comparadas pelos testes F e/ou Tukey adotando-se os níveis de 1 e 5\% de probabilidade. Para as variáveis freqüência respiratória e temperatura retal, o experimento foi analisado segundo um esquema de parcelas subdivididas, tendo nas parcelas um esquema fatorial $3 \times 2$ e, nas subparcelas, os turnos $(\mathrm{T}) \mathrm{de}$ coleta (manhã e tarde), em delineamento em blocos casualizados, com quatro repetições. Na análise de variância, independentemente da significância da interação $\mathrm{T} \times$ grupo $\times$ fibra, optou-se por seu desdobramento. As análises 
estatísticas foram realizadas utilizando-se o software SAEG (Euclides, 1983).

\section{Resultados e Discussão}

Não houve interação grupos genéticos (7/8HZ, 15/16HZ e HPC) × níveis de fibra na ração (30 e 60\% de FDN), mas houve efeito $(\mathrm{P}>0,05)$ dos períodos experimentais sobre $\mathrm{o}$ consumo e o desempenho dos animais. Os consumos de MS, FDN e FDA, expressos em kg/dia e em função do peso corporal (\%PC e g/kgPC ${ }^{0,75}$ ), foram maiores $(\mathrm{P}<0,05)$ nos animais que receberam a dieta FDN60, que, apesar de terem consumido, em valores absolutos, maior quantidade de $\mathrm{PB}$, não apresentaram diferença $(\mathrm{P}>0,05)$ para esta variável.

O maior consumo de MS $(\mathrm{P}<0,05)$ para os animais que receberam a dieta com $60 \%$ de FDN não foi suficiente para que atingissem ganho de peso semelhante aos daqueles que receberam a dieta FDN30. É provável que a inferioridade do ganho de peso diário $(\mathrm{P}<0,05)$ dos animais que receberam a dieta FDN60 esteja relacionada à menor densidade energética da ração, uma vez que, de acordo com o NRC (2001), o percentual de PB das rações experimentais não seria limitante para os desempenhos obtidos em ambos os tratamentos. O consumo de MS nos dois níveis de fibra foi maior que o predito pelo NRC (1989, 2001). Conseqüentemente, os ganhos de peso médios diários foram mais elevados que o esperado.

Embora os resultados não permitam inferir que o consumo da dieta FDN60 foi limitado por mecanismos físicos de controle, de acordo com Resende et al. (1994), a ingestão voluntária de rações com níveis de FDN acima de $50 \%$ é, em geral, regulada por mecanismos de limitação física de consumo.

Avaliando o efeito de diferentes níveis de FDN (40, 50 e $60 \%$ ) sobre o consumo de MS e o crescimento de novilhas da raça Holandesa, Tomlinson et al. (1991), obtiveram consumo máximo em rações com $41 \%$ de FDN. Apesar de os autores não terem separado totalmente os efeitos da FDN e da energia, o nível de fibra teve maior influência sobre o desempenho de novilhas com menos de $200 \mathrm{~kg}$ de peso corporal, em razão da limitação física da capacidade de consumo. Posteriormente, Mertens (1997) acrescentou que essa limitação ocorreria independentemente do tipo de forragem utilizada. Também West et al. (1999), testando quatro níveis de FDN $(30,2 ; 33,8 ; 37,7$ e 42,0) em rações para vacas em lactação, observaram redução linear no consumo de MS, em resposta ao aumento do nível de fibra.

Quigley et al. (1986a;b) observaram, em novilhas leiteiras após a puberdade, que o consumo máximo de MS (8,6 kg/ dia) ocorreu quando as rações em mistura completa apre-
Tabela 3 - Consumos médios e ganho de peso médio diário em novilhas leiteiras recebendo dietas com diferentes níveis de fibra

\begin{tabular}{|c|c|c|c|}
\hline Item & FDN30 & FDN60 & CV (\%) \\
\hline & \multicolumn{2}{|c|}{ Matéria seca } & \\
\hline $\mathrm{kg} / \mathrm{dia}$ & $10,13 b$ & $10,68 \mathrm{a}$ & 8,76 \\
\hline$\%$ PC & $2,70 \mathrm{~b}$ & $2,90 \mathrm{a}$ & 7,75 \\
\hline \multirow[t]{2}{*}{$\mathrm{g} / \mathrm{kgPC}^{0,75}$} & $118,46 b$ & $126,91 \mathrm{a}$ & 2,87 \\
\hline & \multicolumn{2}{|c|}{ Fibra em detergente neutro } & \\
\hline $\mathrm{kg} / \mathrm{dia}$ & $2,70 \mathrm{~b}$ & $6,17 \mathrm{a}$ & 10,29 \\
\hline$\%$ PC & $0,71 b$ & $1,67 \mathrm{a}$ & 7,33 \\
\hline \multirow[t]{2}{*}{$\mathrm{g} / \mathrm{kgPC}^{0,75}$} & $45,45 b$ & $59,13 a$ & 23,10 \\
\hline & \multicolumn{2}{|c|}{ Fibra em detergente ácido } & \\
\hline $\mathrm{kg} / \mathrm{dia}$ & $1,45 b$ & $2,92 \mathrm{a}$ & 10,43 \\
\hline$\%$ PC & $0,38 b$ & $0,79 a$ & 7,89 \\
\hline \multirow[t]{2}{*}{$\mathrm{g} / \mathrm{kgPC}^{0,75}$} & $22,73 b$ & $28,85 a$ & 20,41 \\
\hline & \multicolumn{2}{|c|}{ Proteína bruta } & \\
\hline $\mathrm{kg} / \mathrm{dia}$ & $1,64 \mathrm{a}$ & $1,70 \mathrm{a}$ & 12,62 \\
\hline$\%$ PC & $0,44 a$ & $0,46 a$ & 11,97 \\
\hline \multirow[t]{2}{*}{$\mathrm{g} / \mathrm{kgPC}^{0,75}$} & $18,97 a$ & $20,55 a$ & 17,45 \\
\hline & \multicolumn{2}{|c|}{ Ganho de peso } & \\
\hline $\mathrm{kg} / \mathrm{dia}$ & $1,28 \mathrm{a}$ & $0,92 b$ & 32,27 \\
\hline
\end{tabular}

Médias seguidas de letras diferentes na linha diferem $\left(P<0,05 ;{ }^{*} P<0,01\right)$ pelo teste $F$

sentavam níveis de FDN de 40 a 44\%, valores ligeiramente superiores aos 32 a $40 \%$ de FDN encontrados por Mertens (1989). Os dados encontrados neste trabalho corroboram os obtidos por Quigley et al. (1986a;b), de 1,0 a 1,2 kg/dia, para animais com peso superior a $300 \mathrm{~kg}$. Ganhos de peso de $1,2 \mathrm{~kg} /$ dia em novilhas leiteiras consumindo 9,2 kg/dia de MS/dia também foram observados por Sejrsen \& Purup (1997).

Os consumos de MS, FDN, FDA e PB dos animais HPC, quando expressos em \%PC, foram superiores $(\mathrm{P}<0,05)$ aos observados nos animais 7/8 HZ (Tabela 4), o que reflete a maior capacidade de consumo daquele grupo genético. Os animais do grupo 15/16 HZ apresentaram valores intermediários de consumos de FDN, FDA e PB, que não diferiram daqueles dos demais grupos $(\mathrm{P}>0,05)$.

$\mathrm{O}$ ganho de peso não diferiu $(\mathrm{P}>0,05)$ entre os grupos genéticos e foi em média de $1,1 \mathrm{~kg} /$ dia.

A temperatura ambiente (entre 18 e $26^{\circ} \mathrm{C}$ ) apresentou alta correlação com o ITU $(0,99)$ e o ITGU $(0,94)$ medidos durante o período experimental (Figura 1). O ITU manteve-se acima de 70 no período de 9 às $19 \mathrm{~h}$, acima de 73 no período entre 13 e $15 \mathrm{~h}$ e apenas em alguns momentos ultrapassou a faixa de 74. A umidade relativa do ar permaneceu alta, com média de $87 \%$ durante todo experimento.

Segundo Nääs (1998), o ITU é o parâmetro mais utilizado para avaliação do estresse térmico e, apesar da 
Tabela 4 - Consumos médios e ganho de peso médio diário em novilhas leiteiras de diferentes grupos genéticos

\begin{tabular}{|c|c|c|c|c|}
\hline \multirow[t]{2}{*}{ Item } & \multicolumn{3}{|c|}{ Grupo genético ${ }^{1}$} & \multirow[t]{2}{*}{ CV (\%) } \\
\hline & 7/8HZ & 15/16HZ & HPC & \\
\hline & \multicolumn{3}{|c|}{ Matéria seca } & \\
\hline $\mathrm{kg} / \mathrm{dia}$ & $10,37 \mathrm{a}$ & $10,68 a$ & $10,18 \mathrm{a}$ & 8,76 \\
\hline$\%$ PC & $2,65 b$ & $2,80 a b$ & $2,94 a$ & 7,75 \\
\hline \multirow[t]{2}{*}{$\mathrm{g} / \mathrm{kgPC}^{0,75}$} & $117,70 \mathrm{~b}$ & $123,71 \mathrm{a}$ & $126,64 a$ & 2,87 \\
\hline & \multicolumn{3}{|c|}{ Fibra em detergente neutro } & \\
\hline $\mathrm{kg} / \mathrm{dia}$ & $4,46 a$ & $4,55 a$ & $4,28 a$ & 10,29 \\
\hline$\%$ PC & $1,13 b$ & 1,19ab & $1,25 \mathrm{a}$ & 7,33 \\
\hline \multirow[t]{2}{*}{$\mathrm{g} / \mathrm{kgPC}^{0,75}$} & $50,46 a$ & $52,69 a$ & $53,72 \mathrm{a}$ & 23,10 \\
\hline & \multicolumn{3}{|c|}{ Fibra em detergente ácido } & \\
\hline $\mathrm{kg} / \mathrm{dia}$ & $2,20 \mathrm{a}$ & $2,25 a$ & $2,11 \mathrm{a}$ & 10,43 \\
\hline$\%$ PC & $0,56 \mathrm{~b}$ & 0,59ab & $0,61 \mathrm{a}$ & 7,89 \\
\hline \multirow[t]{2}{*}{$\mathrm{g} / \mathrm{kgPC}^{0,75}$} & $24,91 b$ & $26,09 \mathrm{ab}$ & $26,37 a$ & 20,41 \\
\hline & \multicolumn{3}{|c|}{ Proteína bruta } & \\
\hline $\mathrm{kg} / \mathrm{dia}$ & $1,65 a$ & $1,72 \mathrm{a}$ & $1,64 \mathrm{a}$ & 12,62 \\
\hline$\% \mathrm{PC}$ & $0,42 b$ & $0,45 a b$ & $0,48 a$ & 11,97 \\
\hline \multirow[t]{2}{*}{$\mathrm{g} / \mathrm{kgPC}^{0,75}$} & $18,74 b$ & $20,02 \mathrm{a}$ & $20,51 \mathrm{a}$ & 17,45 \\
\hline & \multicolumn{3}{|c|}{ Ganho de peso } & \\
\hline kg/dia & $1,14 \mathrm{a}$ & $1,10 \mathrm{a}$ & $1,07 a$ & 32,27 \\
\hline
\end{tabular}

Médias seguidas de letra diferentes na linha diferem $(P<0,05)$ pelo teste Tukey.

${ }^{1} \mathrm{HZ}=$ Holandês-Zebu; HPC = Holandês puro por cruza

grande variação segundo os diversos autores quanto aos limites de conforto e estresse, há unanimidade de que ITU acima de 72 é limitante para vacas de alta produção. Entretanto, para novilhas não-lactantes e não-gestantes, é possível que o valor de ITU igual a 72 não caracterize condição de estresse.
De acordo com McDowell (1972), bovinos de todas as idades apresentam graus mensuráveis de desconforto térmico com o ITU igual ou superior a 72, embora o desconforto se torne agudo apenas em índices acima de 78. Os maiores valores de freqüência respiratória $(\mathrm{P}<0,01)$ foram observados no turno da tarde, para ambas as dietas, exceto para o grupo genético 7/8HZ recebendo a dieta com menor nível de fibra (Tabela 5). Os valores encontrados por Martello et al. (2004), em torno de 65 movimentos respiratórios por minuto, foram mais altos que os observados neste experimento, o que pode ser explicado pelos maiores valores de ITU (entre 75 e 76) observados por esses autores.

A temperatura retal situou-se nos limites considerados normais, conforme observado em outros trabalhos (Aguiar et al., 1996; Brosh et al., 1998; Damasceno et al., 1998). As médias das temperaturas retais da tarde foram maiores $(\mathrm{P}<0,01)$ que as da manhã para todos os tratamentos (Tabela 5). Em geral, bovinos apresentam temperaturas retais mais altas no turno da tarde em relação ao período da manhã (Lefcourt \& Adams, 1996; Cunha et al., 2007). Apesar de não terem encontrado diferença significativa em valores absolutos, Martello et al. (2004) também observaram maior temperatura retal de novilhas à tarde $\left(39,3^{\circ} \mathrm{C}\right)$ em relação à manhã $\left(38,3^{\circ} \mathrm{C}\right)$.

As novilhas HPC e as 15/16 HZ que consumiram a ração com $60 \%$ de FDN apresentaram maior $(\mathrm{P}<0,05)$ diferença entre os valores de temperatura retal medidos à tarde e pela manhã, o que pode indicar menor capacidade de dissipação de calor por esses animais. Apesar disso, os dados de temperatura retal e freqüência respiratória obtidos neste experimento indicam que, para as condições de ambiente térmico a que os animais estavam submetidos, as formas latentes de perdas de

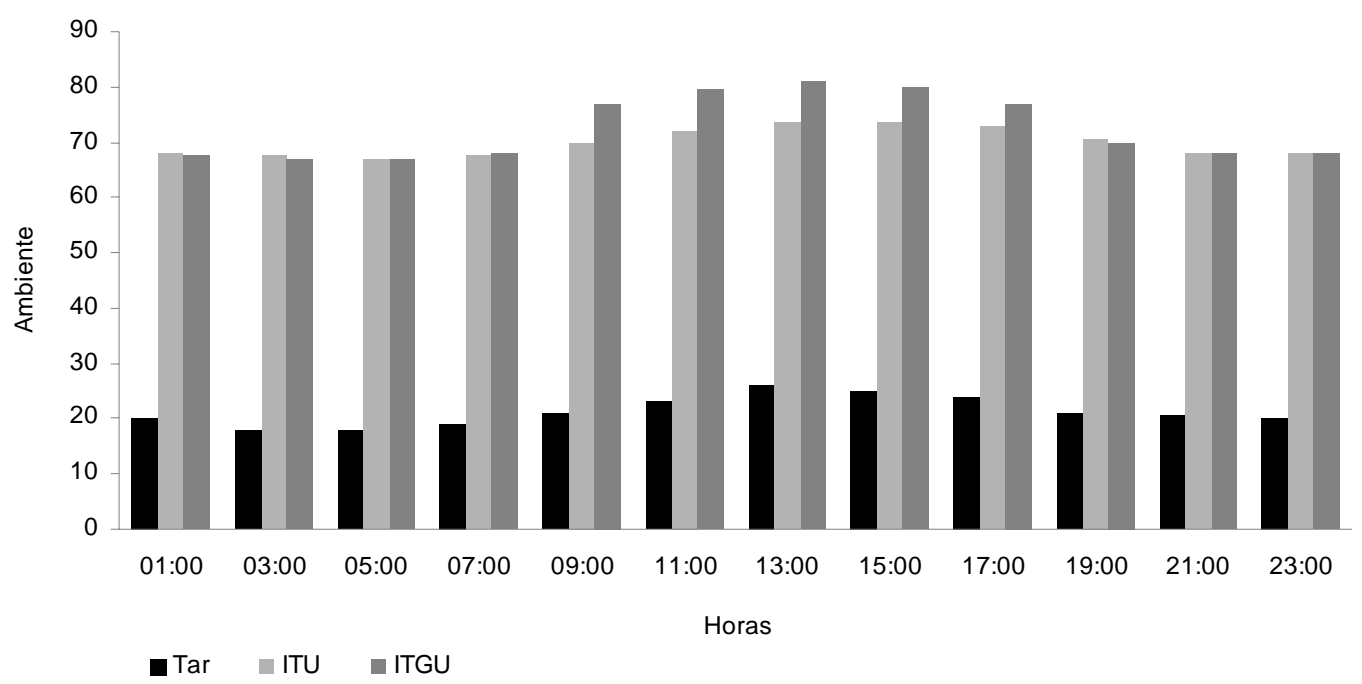

Figura 1 - Temperatura do ar (Tar), índices de temperatura e umidade (ITU) e de temperatura de globo negro e umidade (ITGU), de acordo com a hora do dia. 
Tabela 5 - Valores médios da freqüência respiratória e da temperatura retal em novilhas de três grupos genéticos recebendo dietas com diferentes níveis de fibra

\begin{tabular}{|c|c|c|c|c|c|c|}
\hline \multirow[t]{3}{*}{ Item } & \multicolumn{6}{|c|}{ Grupo genético $^{1}$} \\
\hline & \multicolumn{2}{|c|}{ 7/8 HZ } & \multicolumn{2}{|c|}{$15 / 16 \mathrm{HZ}$} & \multicolumn{2}{|c|}{ HPC } \\
\hline & FDN30 & FDN 60 & FDN 30 & FDN 60 & FDN 30 & FDN 60 \\
\hline Manhã & $31,58 \mathrm{aA}$ & $28,94 \mathrm{bA}$ & $33,58 \mathrm{bA}$ & $33,14 \mathrm{bA}$ & $39,67 \mathrm{bA}$ & $31,00 \mathrm{bB}$ \\
\hline Tarde & $34,92 \mathrm{aA}$ & $36,58 \mathrm{aA}$ & 38,83aA & $39,00 \mathrm{aA}$ & $45,17 \mathrm{aA}$ & $40,97 \mathrm{aA}$ \\
\hline Tarde & $38,95 \mathrm{aA}$ & $38,95 \mathrm{aA}$ & $38,96 \mathrm{aA}$ & $39,10 \mathrm{aA}$ & $39,19 \mathrm{aA}$ & $39,14 \mathrm{aA}$ \\
\hline Tarde-Manhã & $0,44 \mathrm{~B}$ & $0,45 B$ & $0,44 \mathrm{~B}$ & $0,53 \mathrm{~A}$ & $0,57 \mathrm{~A}$ & $0,50 \mathrm{~A}$ \\
\hline
\end{tabular}

Médias seguidas de letra diferente minúscula na coluna e maiúscula na linha, para cada grupo genético, diferem $(P<0,05)$ pelo teste $F$.

${ }^{1} \mathrm{HZ}=$ Holandês-Zebu; HPC = Holandês puro por cruza.

calor foram suficientes para que mantivessem a temperatura corporal nos limites considerados normais.

Nääs (1998) atribuiu a variação da temperatura retal ao longo do dia ao acúmulo de calor no organismo animal, como resultado do excesso de calor recebido do ambiente somado à produção de calor interna durante o dia e à incapacidade dos mecanismos termorreguladores em eliminar esse excedente. Assis et al. (1977), ao avaliarem as reações fisiológicas de novilhas 1/4 HZ, 3/4 HZ e holandesas puras, relataram também que essas variações diurnas da temperatura retal diferem das variações estacionais, uma vez que a aclimatação estacional é regulada por uma mudança no ponto fixo de regulação da temperatura central do animal.

Outra evidência de que os animais não estavam em estresse térmico foi o alto consumo de MS. Segundo West (2003), quando bovinos são expostos a estresse por calor, há redução no consumo de MS, o que não foi observado.

As novilhas 7/8 e 15/16 HZ não diferiram ( $P>0,05)$ quanto à freqüência respiratória. Entretanto, as novilhas HPC apresentaram maior $(\mathrm{P}<0,05)$ freqüência respiratória pela manhã quando receberam a dieta com $30 \%$ de FDN, o que, provavelmente, está relacionado ao maior consumo de energia e à maior geração de calor dos animais que receberam a dieta com 30\% de FDN.

Nogueira (1982), estudando as reações fisiológicas em novilhos de três graus de sangue Holandês-Zebu alimentados com rações com diferentes proporções de concentrado:volumoso, não encontrou efeito sobre a temperatura retal. Entretanto, os animais recebendo a dieta com menor porcentagem de volumoso apresentaram valores de freqüência respiratória significativamente menores. Segundo Barcelos (1984), rações mais fibrosas deveriam proporcionar maiores médias de freqüência respiratória e temperatura retal, uma vez que a maior ingestão de alimentos volumosos aumenta a produção de acetato no rúmen, proporcionalmente ao propionato e ao butirato, resultando em maior produção de calor metabólico.

De acordo com McDowell (1972) e Olbrich et al. (1972), as respostas fisiológicas dos bovinos às variações de temperatura do ambiente diferem em razão de fatores como o nível e o estádio de produção, a proporção de volumosos na dieta e as amplitudes de variação da temperatura ambiente, dos índices de conforto térmico e da umidade relativa do ar. As raças indianas, diferentemente das taurinas não adaptadas, possuem mecanismo de termorregulação mais eficiente e podem perder calor pela sudorese de forma mais efetiva, pois possuem maior número de glândulas sudoríparas ou maior volume de secreção, pêlos mais curtos e maior superfície em relação à massa corporal.

É possível que, durante o período de coleta de dados, nas condições deste experimento, as variáveis de ambiente não tenham sido estressantes o suficiente para ocorrer interação dieta $\times$ freqüência respiratória ou temperatura retal. Os dados da literatura (Harris, 1992; Du Preez, 1990a,b; Baccari Jr., 1998; Damasceno et al., 1998; Nããs, 1998) são, em sua maioria, com vacas leiteiras no início da lactação, no entanto, sabe-se que esses animais apresentam produção de calor metabólico superior à de categorias não-lactantes, em geral, pouco estudadas.

\section{Conclusões}

Quando o capim-elefante é utilizado como único volumoso, novilhas recebendo dietas com $60 \%$ de FDN consomem maior quantidade de MS, FDN e FDA e ganham menos peso por dia em relação àquelas recebendo dietas com $30 \%$ de FDN. Novilhas HPC consomem maior quantidade de MS, em \%PC, em comparação às 7/8 HZ, tanto em dietas com $30 \%$ de FDN quanto com $60 \%$ de FDN. Nas condições de ambiente térmico estudadas, novilhas 7/8 HZ, 
15/16 HZ e HPC alimentadas com rações com 30 e $60 \%$ de FDN mantêm a temperatura retal nos limites fisiológicos considerados normais.

\section{Literatura Citada}

AGUIAR, I.S.; BACCARI JR., F.; GOTTSCHALK. A.F. et al. Produção de leite de vacas holandesas em função da temperatura do ar e do índice de temperatura e umidade. In: REUNIÃO ANUAL DA SOCIEDADE BRASILEIRA DE ZOOTECNIA, 33., 1996, Fortaleza. Anais... Fortaleza: Sociedade Brasileira de Zootecnia, 1996. p.617-619.

BACCARI JR., F. Adaptação de sistemas de manejo na produção de leite em clima quente. In: SIMPÓSIO BRASILEIRO DE AMBIÊNCIA NA PRODUÇÃO DE LEITE, 1, 1998, Piracicaba. Anais... Piracicaba: Escola Superior de Agricultura Luiz de Queiroz, 1998. p.24-67.

BOND, T.E.; KELLY, C.F. The globe thermometer in agriculture research. Agricultural Engineering, v.36, p.251-260, 1955.

BROSH, A.; AHARONI, Y.; DEGEN, A.A. et al. Effects of solar radiation, dietary energy, and time of feeding on thermoregulatory responses and energy balance in cattle in a hot environment. Journal of Animal Science, v.76, n.10, p.2671-2677, 1998.

BUFFINGTON, D.E.; COLLAZO-AROCHO, A.; CANTON, G.H. et al. Black globe- humidity index (BGHI) as comfort equation for dairy cows. Transaction of the american Society of Agricultural Engineering, v.24, p.711-714, 1981.

CARDOSO, R.C.; VALADARES FILHO, S.C.; SILVA, J.F. et al. Consumo e digestibilidades aparentes totais e parciais de rações contendo diferentes níveis de concentrado em novilhos F1 Limousin x Nelore. Revista Brasileira de Zootecnia, v.29, n.6, p.1832-1843, 2000.

CUNHA, N.F.V.C.; CAMPOS, O.F.; PEREIRA, J.C, et al. Desempenho, variáveis fisiológicas e comportamento de bezerros mantidos em diferentes instalações: época chuvosa. Revista Brasileira de Zootecnia, v.36, n.4, p.1140-1146, 2007 (supl.).

DAMASCENO, J.C.; BACARI JR., F.; TARGA, L.A. Respostas fisiológicas e produtivas de vacas holandesas com acesso à sombra constante ou limitada. Revista Brasileira de Zootecnia, v.27, n.3, p595-602, 1998.

DU PREEZ, J.H.; GIESECKE, W.H.; HATTING, P.J. Heat stress in dairy cattle under Southern African conditions. I. Temperaturehumidity index mean values during the four main seasons. Journal of Veterinary Research, v.57, n.1, p.77-87, 1990a.

DU PREEZ, J.H.; GIESECKE, W.H.; HATTING, P.J. Heat stress in dairy cattle under Southern African conditions. II. Identifications of areas of potential heat stress in dairy heat stress summer by means of observed true and predicted temperature- humidity index mean values, Journal of Veterinary Research, v.57, n.3, p.183-187, 1990b.

EUCLIDES, R.F. Sistema para análises estatísticas e genéticas (Manual de utilização do SAEG). Viçosa, MG: Universidade Federal de Viçosa, 1983. 57p.

HARRIS, B. Hot weather dairying: feeding to combat heat stress. Feed International, p.30-32, 1992.

KÖEPPEN, W. Climatologia. Buenos Aires: Panamericana, 1948. $478 \mathrm{p}$

LEFCOURT, A.M.; ADAMS, W.R. Radiotelemetry measurement of body temperatures of feedlot steers during summber. Journal of Animal Science, v.74, p.2633-2640, 1996.
MARTELLO, L.S., SAVASTANO JR., H.; SILVA, S.L. et al. Respostas fisiológicas e produtivas de vacas holandesas em lactação ssubmetidas a diferentes ambientes. Revista Brasileira de Zootecnia, v.33 , n.1, p181-191, 2004.

McDOWELL, R.E. Bases biológicas de la producción animal en zonas tropicales. Zaragoza: Acribia, 1972. 692p.

MERTENS, D.R. Creating a system for meeting the fiber requirements of dairy cows. Journal of Dairy Science, v.80, p.1463-1481, 1997.

NÄÄS, I.A. Tipologia de instalações em clima quente. In: SIMPÓSIO BRASILEIRO DE AMBIÊNCIA NA PRODUÇÃO DE LEITE, 1., 1998, Piracicaba Anais... Piracicaba: Escola Superior de Agricultura Luiz de Queiroz, 1998. p.146-155.

NATIONAL RESEARCH COUNCIL - NRC. Nutrient requirements of dairy cattle. Washington, D.C.: National Academy of Sciences, 2001. 363p.

NATIONAL RESEARCH COUNCIL - NRC. Nutrient requirements of dairy cattle. Washington, D.C.: National Academy of Sciences, 1989. 157p

NOGUEIRA, F.C.B. Reações fisiológicas de novilhos $7 / 8 \mathrm{HZ}$, $1 / 2 \mathrm{HZ}$ e $7 / 8 \mathrm{ZH}$, aos efeitos de fístula, dieta e clima em Viçosa, MG. Viçosa, MG: Universidade Federal de Viçosa, 1982. 94p. Dissertação (Mestrado em Zootecnia) - Universidade Federal de Viçosa, 1982.

OLBRICH, S.E.; MARTZ, F.A.; JOHNSON, H.D. et al. Effect of constant ambient temperatures of $10^{\circ} \mathrm{C}$ and $31^{\circ} \mathrm{C}$ on ruminal responses of cold tolerant and heat tolerant cattle, Journal of Animal Science, v.34, p.64-69, 1972.

QUIGLEY, J.D.; JAMES, R.E.; McGILLIARD, M.L. Equations to predict intake of heifers under intensive management - dry matter intake in dairy heifers. Journal of Dairy Science, v.69, p.2855-2868, 1986a.

QUIGLEY, J.D.; JAMES, R.E.; McGILLIARD, M.L. Factors affecting intake of heifers under intensive management - dry matter intake in dairy heifers. Journal of Dairy Science, v.69, p.2855-2868, 1986b.

RESENDE, F.D.; QUEIROZ, A.C.; OLIVEIRA, J.C. et al. Bovinos mestiços alimentados com diferentes proporções de volumoso:concentrado. 1. Digestibilidade aparente dos nutrientes, ganho de peso e conversão alimentar. Revista Brasileira de Zootecnia, v.30, n.1, p261-269, 2001.

SEJRSEN, J.; PURUP, S. Influence of prepubertal feeding level on milk yield potential of dairy heifers: a review. Journal of Animal Science, v.75, p.828-835, 1997.

SILVA, D.J.; QUEIROZ, A.C. Análise de alimentos (métodos químicos e biológicos). 2.ed. Viçosa, MG: Universidade Federal de Viçosa. 2002. 235p.

TITTO, E.A.L. Clima: influência na produção de leite. In: SIMPÓSIO BRASILEIRO DE AMBIÊNCIA NA PRODUÇÃO DE LEITE, 1. Anais... Piracicaba: NUPEA, ESALQ, USP, 1998. p.10-23.

TOMLINSON, D.J.; JAMES, R.E.; MCGILLIARD, E.D. Effect of varying levels of neutral detergent fiber and total digestible nutrients on intake and growth of Holstein heifers. Journal of Dairy Science, v.74, p.537-545, 1991.

Van SOEST, P.J.; ROBERTSON, J.B.; LEWIS, B.A. Methods for dietary fiber, neutral detergent fiber, and non starch polysaccharides in relation to animal nutrition. Journal of Dairy Science, v.74, p.3583-3597, 1991.

WEST, J.W.; HILL, G.M.; FERNANDEZ, J.M. et al. Effects of dietary fiber on intake, milk yield, and digestion by lactating dairy cows during cool or hot, humid weather. Journal of Dairy Science, v.82, p.2455-2465, 1999.

WEST, J.W. Effects of heat-stress on production in dairy cattle. Journal of Dairy Science, v.86, p.2131-2144, 2003. 